\title{
FACTORES QUE INFLUYEN EN LA CREACIÓN Y UTILIZACIÓN DE LA JERGA ADOLESCENTE EN TRES COLEGIOS DE LA ZONA DE PUNTARENAS
}

\author{
Factors that influence the creation and utilization of teenage jargon \\ in three high schools in Puntarenas region
}

Jonnathan Salas Alvarado*

\begin{abstract}
RESUMEN
La jerga adolescente al igual que cualquier otra variante lingüística responde a una serie de aspectos tales como el género, la ubicación geográfica, la educación, el estatus social, entre otros. Todos estos aspectos hacen que poco a poco los diferentes grupos sociales compuestos por hablantes con características comunes elaboren su propio vocabulario, el cual se espera que les dé identidad y les ayude a diferenciarse de los demás y de la lengua estándar. Los adolescentes están en un momento de sus vidas en el cual les es necesario separarse o diferenciarse de los adultos, esta es una característica propia de la contracultura en la que se incluyen muchos y muchas de ellas. Todo lo que está a su alrededor les afecta y a la larga ocasiona modificaciones en su forma de ser, de vestir, de pensar o de hablar. Esta investigación busca presentar la percepción de los y las estudiantes de undécimo año del Colegio Laboratorio del CUP, el Liceo Emiliano Odio Madrigal y el Colegio Técnico Profesional de Esparza acerca del lenguaje adolescente, las razones que ellos y ellas consideran lo originan y hacen que se distribuya. Esta población participa en un proyecto de investigación realizado durante el año 2015 en cada una de las instituciones ya mencionadas.
\end{abstract}

Palabras clave: Jerga, adolescentes, factores, Puntarenas, léxico.

\begin{abstract}
Teenage jargon like any other linguistic variation responds to a number of issues such as gender, geographical location, education, social status, among others. All these aspects gradually make different social groups that are composed by speakers who share common characteristics develop their own vocabulary. This vocabulary is expected to provide them with a sense of identity and to help them to differentiate themselves from others and the standard language. Teenagers are at a time of their lives in which they need to separate or differentiate themselves from adults, this is a feature of the counterculture in which many of them are included. Everything that surrounds them affects them and eventually causes changes in their way of being, the way they dress, think or speak. This research aims to present the perception of the students of eleventh year of Colegio Laboratorio del CUP, the Liceo Emiliano Odio Madrigal and Colegio Técnico Profesional de Esparza regarding teen language, the reasons they consider originate it and distribute it. This population is involved in a research project conducted during 2015 in each of the aforementioned institutions.
\end{abstract}

Key Words: Jargon, adolescents, factors, Puntarenas, lexicon.

Universidad de Costa Rica. Máster en Ciencias de la Educación con Énfasis en la Enseñanza del Inglés. Profesor e investigador en la Sede del Pacífico. Correo electrónico: josa325@hotmail.com Recepción: 3/1816. Aceptación: 13/9/16. 


\section{Introducción}

La adolescencia es un proceso por el cual pasan todos los seres humanos que llegan a una edad adulta. Esta es una etapa llena de cambios sustanciales para quienes la viven. Durante este periodo se busca afirmar la identidad de cada persona, sin embargo, el lograr definir esta no es fácil, ya que para hacer esto se debe pasar por cambios de todo tipo entre los que destacan los físicos, sexuales y psicológicos.

Parte esencial de la etapa de la adolescencia se desarrolla en el colegio, este es un lugar en el cual los y las jóvenes van a convivir día a día con personas que están pasando por los mismos procesos y que van a tener una empatía mayor hacía ellos, ya que comparten características; es mediante esta interacción que se forman los grupos de pares que son vitales en la construcción y desarrollo de la personalidad de los y las adolescentes.

Uno de los fenómenos más interesantes que logran desarrollar los grupos de jóvenes de colegio es su jerga. La jerga adolescente está compuesta por una serie de palabras y frases que se crean dentro de estos grupos y que van a ser utilizadas durante un tiempo determinado y luego van a ser puestas en desuso y serán sustituidas por otras. Algunas, muy pocas de hecho, se quedan y forman parte del léxico de un grupo de personas, incluso, en algunos casos, estas palabras salen de los grupos de adolescentes y se instalan en comunidades lingüísticas más extensas.

La jerga adolescente se produce por diversas razones, entre ellas, el deseo de diferenciarse de los grupos de adultos, es una parte del proceso de rebeldía hasta cierto punto. Los y las jóvenes no desean ser comprendidos por personas que no forman parte de su círculo de amigos o compañeros, de tal forma que pueden mantener cierta información en secreto. La distribución de la jerga se da mediante varias formas entre las que destacan la interacción, la influencia y uso de medios digitales.

\section{Marco teórico}

\subsection{Adolescencia}

La adolescencia es quizás una de las etapas más cruciales para los seres humanos, puesto que es durante este periodo en el cual muchas de las personas logran formar su personalidad e identidad y definen con ello mucho de lo que van a perseguir en el futuro, "el adolescente disfruta de amplias posibilidades respecto a las elecciones de pareja, el alcance de su educación, vocación y lugar de residencia" (Grinder, 1976: 237).

Según Arroyo, Huertas, Peinaro y Pérez (2014), este periodo de la vida humana es muy complejo debido a las transformaciones de tipo físicas, emocionales, conductuales, intelectuales y sociales que el adolescente conlleva. Una de las tareas más importantes de toda persona adolescente es sin duda la construcción de su propia identidad, para lograr este proceso, el o la joven deben "desligarse de los vínculos que la mantienen cerca del padre y la madre" (Arroyo et al, 2014: 4). En pro de alcanzar esta separación, se necesita experimentar diferentes roles, lograr relacionarse con otras personas, preferiblemente de su edad, y es en este punto en donde cobran importancia los grupos de pares, estos grupos, continúan las autoras, son muy significativos, pues la o el adolescente considera que estos son los únicos que pueden comprenderle y que comparten puntos de vista similares, y es esta la razón por la cual las personas adolescentes tienden a distanciarse de sus padres, a quienes consideran anticuados, y se aferran a otros que se parezcan más a ellos. En esta misma línea y en relación con la relevancia de los grupos de pares, Grinder (1976) menciona que "el adolescente buscará apoyo emotivo entre sus camaradas porque su familia no lo ayuda a alcanzar la madurez plena social y vocacional" (p. 240), parece ser que son los grupos de pares los que van a ayudar a los y las adolescentes a lograr una efectiva transición hacia la adultez, ya que es mediante estos y la interacción que se logra que los y las jóvenes definan una 
personalidad más independiente alejada del control familiar.

Aguilar, Pérezy Víquez(2007) concuerdan con lo anteriormente expuesto al considerar que la persona adolescente debe llevar a cabo "la búsqueda y concreción de una identidad personal y social a partir de la experimentación y el cotejo de diferentes formas de vida, roles e ideologías" (p. 44); sin embargo, estos mismos autores a la vez mencionan el hecho de que este es un periodo de transición hacia la adultez y, como tal, implica crisis de tipo psicológicas que pueden ser pasajeras, además de ello, en esta etapa los y las jóvenes deben reflexionar sobre su futuro y deben resolver todo lo referente a la dependencia familiar.

De acuerdo con un estudio realizado por Aguilar et al (2007), se llega a una serie de características que pueden referir a pautas conductuales adolescentes:

a) la tendencia a la exploración y a la experimentación de nuevos espacios y acciones; b) el descontrol; c) la integración y la identificación con el grupo de pares; d) y la consecuente separación y progresiva independencia respecto a la pareja parental (p. 51).

Por otra parte, este mismo estudio brinda otra serie de características de la población adolescente, a saber:

Caracterizados por la anomia, la falta de controles y el cuestionamiento de las relaciones sociales (familiares y societales) así como por el desvío respecto a las normas que las rigen. Los padres ya no son el eje fundamental de referencia, sino que se amplía a los pares, existiendo un desplazamiento hacia este grupo (Aguilar et al, 2007: 51).

Finalmente, es importante mencionar a Krauskopf (2006) quien hace un breve resumen de los procesos y adquisiciones principales que deben alcanzar los y las adolescentes en nuestra cultura occidental para lograr culminar esta etapa de manera exitosa: la sexualidad que debe establecerse y definirse, el esquema corporal que debe formarse después de los cambios físicos por los que pasa la persona adolescente, estos cambios deben ser asimilados para afirmar el atractivo y la aceptación sexual.
La autoestima es otra de las metas a alcanzar y esta evoluciona, según la autora, desde la valorización de los padres hasta ser afirmada mediante propias capacidades. Otros aspectos que se deben lograr son las destrezas intelectuales y la individuación misma en la que, según lo establece Krauskopf, "se enfatiza fuertemente el sentido de sí mismo, acelerando el proceso de desimbiotización" (2006: 51). Además, se debe alcanzar la autonomía y la identidad que se deben desarrollar a lo largo de esta etapa de la vida. Por otra parte, se logra conseguir una participación social y un proyecto de vida, la primera se consigue mediante la revisión del propio esquema de creencias y valores $\mathrm{y}$, el segundo, evoluciona con el tiempo. Finalmente, se busca una personalidad.

\subsection{Adolescentes, la contracultura y los medios de comunicación}

La adolescencia es un período caracterizado por muchos aspectos particulares, uno de ellos es sin duda la rebeldía. La rebeldía en realidad no es fortuita, es un momento de la vida en el cual los y las jóvenes construyen su identidad y parte de este proceso consiste en rechazar paradigmas y normas existentes.

La contracultura es un movimiento que se opone a las normas y no es un hecho ni aislado ni mucho menos reciente. Inicia cerca de los años 60 , en los cuales la juventud toma un rol más preponderante oponiéndose a muchas doctrinas sociales, otorgándose privilegios y proponiendo cambios importantes. Este movimiento, según señala Rodríguez (2006) obedece entonces a la ruptura de "un orden y unos valores morales en los que no creían" (p. 8), y es debido a esto que los y las jóvenes crean su propia cultura, dicha cultura, continúa el autor, se caracteriza en ese momento por varios aspectos tales como un "un énfasis en la música rock, las drogas psicodélicas, las comunas y la filosofía oriental y hermética" (Rodríguez, 2006: 8).

Ese movimiento, con el paso de los años, ha generado la aparición de un sinfín de grupos o subculturas que han mantenido un norte en común: el rechazo de una cultura estandarizada 
que todos y todas deberían de seguir. Muchos de esos grupos son iniciados y se nutren a través de los años de personas jóvenes, en muchos casos, visionarias que buscan marcar una clara diferencia e imponerse en contra de los sistemas que los y las gobiernan. Un aspecto muy importante a destacar es el hecho de que todas estas ideas han logrado mantenerse y reproducirse gracias a los medios de comunicación que se han encargado de divulgar $y$, en muchos aspectos, fomentar ciertas actitudes y comportamientos.

Uno de los medios de comunicación masiva que tradicionalmente ha marcado a la sociedad ha sido la televisión, se ha entendido como un agente cultural que tiene la capacidad y en muchas ocasiones la función de socializar a las y los individuos. Pérez (2002) establece que la televisión tiene tres funciones en el proceso de socialización de las personas. La primera de ellas es la contribución que brinda a la construcción de la identidad, otra es la estructuración de la vida cotidiana de las personas y, finalmente, el autor señala que la televisión "contribuye a la formación de competencias televisivas: en el transcurso de la socialización televisiva los sujetos se apropian de las estructuras de narración y representación que caracteriza a cada género televisivo" (p. 9).

Parece ser que la magia de la televisión yace en ese vínculo que se logra entre la imagen y el sonido y con ello el poder de transmitir ideas en tiempo real. García (2004) indica que la televisión es el medio de comunicación de masas "de mayor difusión al no necesitar ninguna preparación previa para su consumo" (p. 118).

Sin embargo, más allá de la televisión que por años ha sido el medio de comunicación por excelencia, actualmente hay otros medios como los teléfonos inteligentes, las redes sociales $y$ el Internet, que en general bombardean constantemente a nuestra sociedad y que han estado ocasionando cambios en ella de manera paulatina. Así por ejemplo, Sádaba (2005) manifiesta que los medios, para la juventud actual, no representan sorpresa alguna, ya que para esta generación siempre han estado ahí. Esta es una generación que ha crecido rodeada por la música, los videojuegos, las computadoras, el internet, los teléfonos celulares, los cuales según ella, "se han convertido en medios familiares para la juventud, donde encuentra los contenidos que le interesan, y sobre todo una herramienta que le permite satisfacer su curiosidad y necesidad de comunicación con sus iguales" (Sábada, 2005: 12).

Otro aspecto que señala Sábada (2005) es el hecho de que los y las adolescentes se aíslan de sus familias en sus habitaciones y es desde ellas que se conectan con el mundo y con sus pares. Las posibilidades tecnológicas con las que cuentan los y las jóvenes actualmente hacen que con solo una computadora con acceso a Internet o un teléfono celular, ya sea inteligente o no, el o la joven va a ser capaz de mantener el contacto con sus amigos a la distancia y desde la privacidad de su cuarto. Según la autora, "los jóvenes utilizan el contenido de los medios para reafirmar o argumentar sus opiniones e ideas, como parte de su proceso de socialización y para desarrollar su sentido de pertenencia: en muchos casos este contenido vehicula las conversaciones de los grupos juveniles" ( $p$. 13), dicho de otra manera, los medios dan un sentido de poder a los y las adolescentes, les posibilitan manifestarse y expresarse.

En lo que respecta a las redes sociales, se puede decir que son un fenómeno reciente pero que han calado profundamente en la sociedad, particularmente en la población más joven. Las redes sociales son básicamente comunidades virtuales a las cuales los y las usuarias se unen e interactúan. Todas poseen una dinámica diferente, pero al final se basan en lo mismo: compartir ideas, pensamientos, opiniones, aspectos personales o familiares e interactuar.

Molina y Toledo (2014) aseguran que las redes sociales han entrado en el mundo de manera rápida $\mathrm{y}$, de la misma forma, han traído una serie de cambios considerables. Este fenómeno "ha ocasionado un profundo impacto en la sociedad actual principalmente en las nuevas generaciones, debido a que ha implantado un nuevo estilo de relacionarse entre los propios jóvenes" (Molina y Toledo, 2014: 1). Como parte de los cambios que mencionan las 
autoras se destaca la comunicación virtual, la cual se puede generar de diversas formas tales como los chats, las videoconferencias y otras. Este tipo de herramientas tecnológicas han revolucionado la manera en que las personas interactúan y les da un sinfín de posibilidades que día tras día aumentan, y esto hace que las redes sociales ganen más adeptos con el paso del tiempo, incluso personas de edad avanzada se han ajustado y son parte activa de los nuevos cambios propuestos por estas herramientas.

Dentro de las redes sociales más populares actualmente vale la pena citar a Facebook, Instragram, Twitter y Snapchat. Facebook nace en el año 2004 y es la red social más popular del mundo con más de 200 millones de usuarios, este es un medio en el cual las personas pueden publicar información personal o profesional, compartir fotos, videos, ser parte de chats, entre otras cosas. Instagram, por su parte, es una red social con tendencia visual que ha logrado tener mucha popularidad. Según explican Molina y Toledo (2014), debido a "la facilidad con la que se puede compartir fotos con diferentes filtros que las hacen más agradables" (p. 20), la idea es compartir fotos a las cuales se les pueden adjuntar comentarios y localización geográfica. Twitter nace en el 2006 y ha venido en constante crecimiento en cuanto a popularidad y, por ende, número de seguidores. La característica más particular de esta red social es que limita a sus usuarios a escribir únicamente 140 caracteres en sus publicaciones (tweets), esta característica ha hecho que en muchos casos las personas que hacen uso de esta red social se vean obligadas a hacer uso de abreviaciones o de acrónimos. Finalmente, Snapchat es una aplicación móvil que nace en Estados Unidos en el 2010 y que ha ido creciendo en popularidad especialmente entre los y las adolescentes. Esta aplicación se basa en una plataforma que permite a los usuarios compartir archivos, fotos y videos (snaps) a los cuales se les puede establecer un tiempo determinado de duración y además se les puede agregar una serie de filtros. Los "snaps" desaparecen después de cierto tiempo y no pueden ser descargados por las personas que los ven y si alguna persona hace una captura de pantalla, el o la dueña de la cuenta será notificada de manera inmediata.

El uso frecuente de redes sociales ha provocado que el lenguaje de sus usuarios se haya venido modificando poco a poco al punto de que nuevas palabras se han acuñado y existe una mayor presencia de anglicismos dentro del español de Costa Rica, tanto así que es muy común escuchar a los y las jóvenes hablar de "tweets","posts" y "snaps" diariamente, aspecto que en algunos casos desconcierta a los y las adultas a su alrededor.

En relación con los teléfonos inteligentes, estos pertenecen a lo que se conoce como comunicación móvil. Ríos (2010) establece que la comunicación móvil es el medio de comunicación más importante a nivel mundial, ya que permiten acortar las distancias y acercar a las personas. Los teléfonos móviles o celulares son, de repente, los ejemplos más claros de este tipo de comunicación. De acuerdo con Ríos (2010), los teléfonos celulares "abarcan todos los ámbitos de la actividad humana (trabajo, la escuela, la familia, fiesta, entretenimiento)" (p. 51). Además de ello, es importante resaltar el hecho de que estos aparatos cumplen con una de las necesidades más importante para los seres humanos: la comunicación. Es mediante este tipo de dispositivos que los seres humanos interactúan entre sí de muchas formas, ya sea mediante las llamadas de voz, las video llamadas, los mensajes de texto o las redes sociales y el internet que se hacen indispensables dentro de los dispositivos inteligentes.

Las personas, en los últimos años, han venido desarrollando una especie de dependencia telefónica que a veces llega a extremos preocupantes. Por ejemplo, si alguien deja el teléfono olvidado en algún sitio, esta persona va a experimentar un sentimiento de preocupación enorme y si el aparato deja de funcionar, en muchos casos, las personas se sienten fuera del mundo, se depende tanto de los teléfonos que "hemos delegado distintas actividades que solíamos realizar (memorizar números telefónicos, direcciones, usar el reloj, etc.) a las funciones que nos posibilita el teléfono celular" (Ríos, 2010: 52). 
En el caso de la juventud existe una gran dependencia a los medios electrónicos y particularmente a los teléfonos inteligentes, puesto que en ellos pueden realizar todo lo que necesitan. Pueden establecer contacto e interactuar con sus seres queridos, compartir ideas, averiguar cosas, enterarse de eventos $\mathrm{y}$ acontecimientos y aprender. Es en estos aparatos en los cuales se van a encontrar muchas de las herramientas necesarias para construir su identidad.

\subsection{Variantes del lenguaje juvenil}

Herrero (2002), quien es citada por Boyer (2013), establece que existen tres tipos de variantes a saber en el lenguaje juvenil: “(1) diastráticas, aludiendo a los factores sociales como la edad, el sexo, el nivel cultural, la clase social, etc.; (2) diafásicas, para la situación y el contexto de uso en los que se produce, y (3) diatópicas, considerando los factores geográficos" (p. 20).

Dentro de las variantes diastráticas podemos comprender la existencia de fenómenos tales como el registro, esto en relación con lo que se puede o no considerar culto, vulgar o estándar según cada grupo social. En cuanto a las variantes de tipo diafásicas entran en escena las jergas, el argot, los niveles coloquiales y formales, de acuerdo con cada situación lingüística. Finalmente, al hablar de las variantes diatópicas, se puede hablar de aspectos como los que se generan cuando dos o más lenguas se encuentran en contacto tales como la diglosia y el bilingüismo.

Zimmer (2004) considera que el habla estudiantil, que para efectos de este estudio será el equivalente al lenguaje juvenil, pertenece o se relaciona con la variante diástrica, ya que esta es "una variedad de un grupo determinado que se usa solamente en ciertas situaciones" ( $p$. 162); sin embargo, la misma autora establece que no se puede dejar de lado la variante diafásica, debido al hecho de que los jóvenes hacen uso de su variante léxica únicamente en contextos informales o cuando se encuentran con sus grupos de amigos.
Es importante mencionar lo que Ríos (2010) establece: en el momento de hacer un análisis del lenguaje juvenil es de suma importancia considerar aspectos de carácter sociocultural y de uso de registro o contexto. De acuerdo con la autora, los y las jóvenes van a variar su registro de acuerdo con su interlocutor. Por otra parte, la autora señala que es posible que existan diferencias lingüísticas a nivel de género, clases sociales y localización geográfica (rural o urbana).

Ríos (2010) cita cuatro factores que Herrero (2002) considera que van a identificar o a caracterizar el lenguaje juvenil:

1. "El tono que domina en la conversación es el informal que se realiza con amigos, con novios, con compañeros de clase, etc.

2. La finalidad interpersonal es clara: los jóvenes hablan para estrechar sus lazos y relaciones.

3. Los temas que tratan pertenecen al ámbito cotidiano, a lo vivencial, a las actividades o inquietudes juveniles como el estudio, las relaciones personales y afectivas, las salidas nocturnas, la música, la ropa, etc.

4. El modo o canal es el oral. Hablan de modo espontáneo y no planificado alternando papeles de emisor y receptor sin estar fijados de antemano. En algunas ocasiones el canal puede ser el escrito pero siempre como manifestación del lenguaje oral" (p. 133).

Mitkova (2009) indica que existen muchos factores sociales que inciden en los usos lingüísticos y que la edad de los hablantes innegablemente es uno de estos, en efecto, según lo establece la autora, esta no actúa por si sola pero sí posee la relevancia suficiente para poder señalar algunas características del habla de los grupos. En el caso del habla juvenil, las autoras consideran que es en donde se pueden observar más rasgos representativos y específicos.

\subsection{Funciones del lenguaje juvenil}

En relación con las funciones sociales del lenguaje juvenil, Boyer (2013) cita a 
Zimmermann (2002) quien considera que este busca la construcción de la identidad juvenil y la identificación con subculturas también jóvenes. Esta función identataria, señala Boyer (2103), se manifiesta "en el habla de los jóvenes por un distanciamiento con la lengua estándar. Para responder a las idiosincrasias de su generación" (p. 24). De acuerdo con Chang y Guadalupe (2011), los hablantes de una lengua tienden a adoptar "el vocablo con una nueva sonoridad para hacerlo más expresivo, y lo dotan de contenido semántico diferente al original" ( $p$. 4). Se entiende entonces que el lenguaje juvenil es un intento de esta población por diferenciarse de otros grupos, por crearse una identidad propia y por distanciarse de normas y reglas impuestas socialmente.

Assman (1993), citado en Zimmer (2004), establece que la identidad se constituye mediante interacción y se basa en el lenguaje. El habla estudiantil, continúa la autora, "le brinda a los estudiantes un marcador intragrupal que hacia afuera los diferencia de los demás grupos, especialmente de los adultos" (p. 162). En términos de identidad, Velázquez (2007) considera que los y las jóvenes se encuentran consolidando esta en términos personales y sociales. El lenguaje "les permite nombrarla y elaborarla" (p. 85), además de ello, la interacción con otras personas les da la posibilidad de "tomar referentes para su diferenciación como un grupo social diferente a los niños y a los adultos, y con una presencia como sujetos o actores sociales" (Velázquez, 2007: 85).

Sánchez y Ramírez (2011) manifiestan que la jerga juvenil "da cohesión de grupo $\mathrm{y}$, principalmente, que sirve como medio de aprehensión de su cultura" (p. 93). Este tipo de lenguaje, continúan los autores, es una señal de rebeldía y contraculturalidad de parte de los y las jóvenes. La jerga, por tanto, "responde a un medio expresivo que da identidad y define a un grupo o comunidad lingüística" (Sánchez y Ramírez, 2011: 93). Podemos decir entonces que estamos ante un fenómeno muy particular que pone en manifiesto la rebeldía adolescente y que esta hace uso del lenguaje para oponerse, de cierta manera, a las normas establecidas y que en parte ellos y ellas sienten que le son impuestas por los adultos.

Ríos (2010), hace mención a Avello y Muñóz (1989) al referirse al hecho de que el lenguaje juvenil es "una reacción a las instituciones como la familia, la escuela, la iglesia y los medios de comunicación de masas que les dicen qué deben hacer y qué no, cómo deben actuar y qué deben ponerse, entre otras cosas" (p. 135). La jerga, continúa la autora, le da identidad a los y las jóvenes solo en tanto sean miembros del grupo que la utiliza.

\subsection{Lengua y género}

Los seres humanos, tal y como se ha mencionado anteriormente, somos diferentes en muchos aspectos. Existen marcadas diferencias en aspectos sociales, generacionales, económicos y de género. En este caso, podemos decir que los hombres y las mujeres manejan un lenguaje diferente aún a pesar de pertenecer a clases sociales o regiones dialectales similares.

Coates (2004), citado en Rundblom (2013), menciona que "las mujeres viven según los ideales que dictan cómo una mujer debe hablar" (p. 9). Sobre esta línea y citando a Lozano (2005) y a Coates (2004), en Rundblom (2013), establecen que existen muchos estereotipos en relación al lenguaje femenino, los cuales, según los autores, se han ido reproduciendo a través de libros, chistes, canciones y otros, de tal forma que se han creado ideas sobre el habla femenina, por ejemplo, que las mujeres hablan demasiado o que hablan suavemente. La diferencia en el lenguaje, según Lozano (2005), se produce debido a la "forma de tratar ambos sexos desde la infancia" (Rundblom, 2013: 9). Ambos sexos reciben un trato distinto desde el día en el que un bebé viene al mundo, se les educa de acuerdo con lo que un hombre o una mujer debe o no debe hacer o decir, la manera en la que debe comportarse o la manera en la que deben hablar o vestir.

Adelswärd (1999), citado en Rundblom (2013), considera que al etiquetar los comportamientos de género se corre el riesgo de que estos se hagan reales. Parece ser que "Las mujeres y los hombres usan el lenguaje de 
manera diferente en función de las diferentes expectativas culturales" (Rundblom, 2013: 9), de tal forma que, dependiendo de cada cultura y de lo que se espera de cada género, los hombres y las mujeres van a ser capaces o no de proferir o de hacer uso de determinadas estructuras o de determinado léxico. De hecho, es interesante que en muchas sociedades el lenguaje se va modificando día tras día y con el paso de los años a las mujeres se les ha permitido hacer uso de ciertas palabras que en el pasado simplemente eran impensables para ellas. Vemos entonces cómo la expectativa social de la mujer se va modificando y esto genera consigo cambios léxicos.

Según Yule (2010), parece ser que dentro del discurso femenino existe la tendencia de invitar al acuerdo, los hombres por su parte tienden a usar formas lingüísticas más asertivas y fuertes. La diferencia del lenguaje masculino y femenino, como apunta Domingo Lozano (2005), citado en Rundblom (2013), es que los hombres tienden a insultar o maldecir con más frecuencia que las mujeres, además de ello, continúa la autora, los hombres tienden a usar palabras más fuertes en tanto que las mujeres prefieren palabras más suaves. Adelswärd (1999), en Rundblom (2013), afirma que "las mujeres son, por ejemplo, más solidarias, sensibles, personales e íntimas en sus conversaciones, mientras que los hombres son más dominantes y se centran en un sistema de estatus jerárquico" (p. 10), esto es sin duda parte de la herencia cultural que le permite a los hombres tener mayor presencia y más poder en casi todos los contextos, en tanto que se deja a la mujer de lado, y el lenguaje que utilizan ambos géneros es un reflejo de ello. Rundblom (2013) además cita a Einarsson (2004), quien asegura que la mujer al estar subordinada al hombre busca un estatus social más alto y hace uso del lenguaje para lograrlo, lo cual es algo a lo que los hombres no le dan importancia y es por ello que tienden a hacer uso de lenguaje más soez que las mujeres.

Por su parte, Ríos (2010) revela que en diferentes estudios en los que se ha considerado la variable de género en cuanto al uso de la lengua, se ha concluido que existen diferencias de tipo fonéticas, morfológicas y léxicas entre hombres y mujeres. Estas diferencias, continúa la autora, se pueden producir debido a diversos aspectos tales como diferencias en la organización cerebral, las relaciones de poder y solidaridad y los aspectos culturales y educacionales. Además, menciona que ciertos términos escogidos por mujeres $u$ hombres no son del todo aleatorios, son más términos marcados. De esta forma, ciertas palabras podrían considerarse exclusivamente femeninas o masculinas según sea el caso. En esta línea, Ríos comenta que nuestra sociedad "orienta a los hombres hacia una conversación de poder, mientras que las mujeres buscan señales de solidaridad" (2010: 250). Basado en esto, la autora afirma que a las mujeres se les ha enseñado a no interrumpir en tanto que a los hombres se les ha enseñado a ser competitivos y a mantener el poder. Dentro de la sociedad, se han ido limitando las posibilidades expresivas de ambos sexos, de tal forma que a los hombres se les ha prohibido manifestar afectividad en su léxico y a las mujeres se les ha censurado todo intento de expresar agresividad mediante insultos u ofensas. A los individuos se les ha limitado la posibilidad de expresar sus sentimientos abiertamente mediante el lenguaje.

\subsection{Lengua, clase social y variedades regionales}

La lengua va a presentar variantes dependiendo de la zona en la que los y las hablantes se encuentren, de tal forma, es muy probable que las personas que habitan en una zona urbana posean variantes lingüísticas considerables en relación con las personas que habitan en zonas más urbanas. De igual forma, la clase social o el estatus socioeconómico de los individuos puede eventualmente ocasionar variantes. De acuerdo con Yule (2010), la existencia de dialectos regionales diferentes es ampliamente reconocida y a menudo es motivo de humor para aquellos que viven en otras regiones, este aspecto humorístico al cual refiere el autor responde directamente a las actitudes lingüísticas que poseen las personas, esa idea de creer que una variante lingüística es mejor 
o peor que otra, darle prestigio a una y obviar o rechazar otras.

Valeš (2009) señala que el prestigio puede determinar "el futuro de las hablas, variedades y las lenguas. En general, las variedades de mayor prestigio tienden a sobrevivir y arrinconar las de menor prestigio" (p. 232). Esto es precisamente lo que sucede en relación con las variedades lingüísticas: existen variedades que cuentan con prestigio otorgado por una comunidad de hablantes y es esa la que se va a mantener, la que los y las hablantes van a reproducir. Pero, al mismo tiempo, las variantes que cuentan con un prestigio bajo van a mantenerse recluidas a ciertas áreas geográficas o grupos específicos, quienes a su vez las verán como poco prestigiosas y eventualmente tratarán de ponerlas en desuso y sustituirlas por las variantes o variante que cuente con un mayor prestigio dentro de la comunidad lingüística. Continuando con el tema, el autor establece que

sería erróneo considerar que los hablantes del nivel sociocultural más alto establecen un estándar de mayor prestigio que todos los demás tienden a imitar. Al contrario, numerosos estudios han comprobado que normalmente son los sectores medios de la sociedad los que originan y promueven los cambios lingüísticos (Valeš, 2009: 233).

Lo anterior está probablemente relacionado con el hecho de que la clase alta no es mayoritaria en ninguna sociedad, de tal forma que es muy poco probable que genere cambios fuertes a nivel lingüístico. Por el contrario, la clase media al más popular tiene la posibilidad de generar y reproducir dichas variantes a mayor escala. Sin embargo, lo cierto del caso es que tal y como apunta Salazar (2015), "un dialecto puede ser considerado "más correcto" que otro sencillamente porque es hablado por un grupo socialmente prestigioso y esa valoración la hacen los mismos hablantes" (p. 44). De tal forma que aun cuando sean las clases medias las que tienen más posibilidades de hacer cambios lingüísticos dentro de los grupos sociales, siempre se va a considerar la variable utilizada por las clases altas como el modelo a seguir o la forma estandarizada de la lengua.
Para comprender más sobre el prestigio lingüístico es importante mencionar que existen dos tipos. Según Yule (2010), el prestigio abierto es un estatus generalmente reconocido como mejor y con una valoración más positiva por parte de la comunidad. El prestigio encubierto, por su parte, posee un estatus escondido que, si bien es cierto, cuenta con el favor de un grupo determinado de hablantes este no lo exhibe. De acuerdo con Valeš (2009), "la variante estándar habitualmente goza de mayor prestigio abierto que las variantes no-codificadas" (p. 233). Las variantes no codificadas o más bien no estándar son menos populares, ya que no dan prestigio a los y las hablantes.

\section{Metodología}

\subsection{Lugar y participantes}

Este estudio se realizó en tres colegios de la región de Puntarenas: el Colegio Laboratorio del CUP (CUP), el Liceo Emiliano Odio Madrigal (LEOM) y el Colegio Técnico Profesional de Esparza (CTPE). El primero es un colegio académico privado que se ubica en el cantón central de la provincia de Puntarenas, es un colegio con más de 25 años de existencia. Esta institución, a pesar de ser privada, se caracteriza por no ser necesariamente cara, de hecho es bastante accesible, y de las instituciones privadas de la zona es la que tiene un menor costo de matrícula y mensualidad. La institución es dirigida por una fundación sin fines de lucro que además promueve el acceso de la educación de calidad a sectores menos favorecidos de la sociedad, de hecho se promueve un sistema de becas para ayudar a los y las estudiantes sobresalientes de la zona que no cuentan con los recursos económicos para costear una educación privada. El Liceo Emiliano Odio Madrigal se localiza en la comunidad de Marañonal en el distrito central del cantón de Esparza, es una institución relativamente nueva, abre sus puertas en el año 2001, además, es un colegio académico público. Finalmente, el Colegio Técnico Profesional de Esparza es una institución que se 
crea en el año 2013 y se ubica en la comunidad de Barón, en el distrito de San Rafael en el cantón de Esparza. Esta es una zona rural y la mayoría de los y las estudiantes que asisten a este colegio son también de zonas rurales del cantón de Esparza. Es una institución de tipo técnica y pública.

El estudio contó con la participación de 66 estudiantes de undécimo año, un grupo de cada colegio, 26 del Colegio Laboratorio del CUP, 23 del Liceo Emiliano Odio Madrigal y 17 del Colegio Profesional de Esparza. Las edades de los y las participantes del estudio van de los 16 a los 18 años.

En el caso de los y las estudiantes del Colegio Laboratorio del CUP, la mayoría vive en el Centro de Puntarenas, el Roble, Miramar y el centro de Esparza. En su caso, la mayoría confiesa que ambos padres trabajan fuera de la casa $(46 \%)$ y las ocupaciones que tienen los padres son, entre otras, abogados, profesores, auditores, enfermeros, ingenieros, administradores de empresas. Las familias a las que pertenecen son en su mayoría pequeñas, de hecho un $29 \%$ de los y las participantes son hijos únicos y un $38 \%$ únicamente tiene un hermano o hermana.

En cuanto al Liceo Emiliano Madrigal, la mayoría de los y las estudiantes vive en Marañonal, La Riviera y el centro de Esparza (poblaciones céntricas). En cuanto a las ocupaciones de los y las encargadas de estos estudiantes se mencionan abogados, maestros, amas de casa, choferes, policías, administradores, operadores de maquinaria, costureras, entre otros. Estas familias cuentan con 4 miembros $(60 \%)$ o más de $4(20 \%)$, solo un $20 \%$ de los participantes son hijos (as) únicos.

Por otra parte, el Colegio Técnico Profesional de Esparza cuenta con una población que habita en comunidades como El Barón, San Juan, Nances, Santa Marta, entre otras, todas las mencionadas son de corte rural. Las ocupaciones de las personas encargadas de estos estudiantes son en su mayoría amas de casa, técnicos, electricistas, pulperos, peones, empleadas domésticas, pescadores, constructores, mecánicos, profesores y maestras.
Las familias de estos jóvenes son en su mayoría de 3 miembros (40\%) o de más de 4 personas (40\%); un dato muy interesante en este caso es que en $47 \%$ de los casos únicamente una persona del núcleo familiar trabaja, y considerando las ocupaciones que enlistan los y las participantes, podemos pensar que se trata de una población con posibilidades económicas un poco más limitadas que en los otros dos colegios.

\subsection{Procedimiento investigativo}

Este trabajo es el resultado de un proceso investigativo de varios meses como parte de las actividades de un proyecto de investigación inscrito en la Sede del Pacífico de la Universidad de Costa Rica. Para conseguir los resultados que se presentan en este documento, se hace un conversatorio con los y las estudiantes de los 3 colegios. Se separan a los hombres y a las mujeres y se realiza un conversatorio semiestructurado en el cual se tocan temas relacionados con la jerga adolescente, la percepción que ellos y ellas tienen, su percepción acerca del lenguaje adulto, el lenguaje rural y urbano, las diferencias lingüísticas con respecto al género y las razones por las cuales ellos y ellas hacen uso de ciertas palabras o expresiones. Posterior a ello, se elabora y aplica un instrumento a la totalidad de la población participante, con lo que busca conseguir información acerca de la familia (cantidad de personas, ocupación de los padres y madres de familia), el uso de medios tecnológicos y el origen de las frases y palabras pertenecientes a la jerga adolescente que forman parte de su léxico.

\section{Análisis de resultados}

Los datos obtenidos de esta investigación serán analizados con base en las variables más relevantes que inciden en la creación, distribución y reproducción de la jerga juvenil, así como en la percepción que tienen los y las participantes de su lenguaje en relación con el de las personas de otras edades, género y lugar de residencia. 


\subsection{Influencia de los medios de comunicación en el origen y distribución de la jerga adolescente}

Una de las hipótesis que cobra más fuerza en lo que se refiere al origen y reproducción de la jerga juvenil es el del uso de los medios tecnológicos tales como la televisión, la radio, el internet y las redes sociales. Ante esto, se hace la consulta a los y las participantes con respecto a la tenencia y al uso del teléfono celular, en donde un $100 \%$ de la población entrevistada dice tener un teléfono celular propio, el cual, según confiesan los y las estudiantes, es usado en su mayoría para navegar en Internet y hacer uso de redes sociales.

Con respecto a la frecuencia con la que los y las participantes del estudio hacen uso de su teléfono celular, tal y como se puede apreciar en la tabla 1, en el caso del CUP un $71 \%$ dice hacer uso del aparato celular siempre, en tanto que los y las estudiantes del LEOM, un 73\% dice usarlo siempre. El caso del CTPE baja considerablemente, ya que solo un $47 \%$ dice hacer uso del celular siempre. Sin embargo, en todos los casos los números se mantienen en el "casi siempre" y el "siempre", en muy pocos casos se presenta un "a veces", por tanto, tenemos una población que está muy relacionada con el uso del teléfono celular; este aparato tecnológico forma parte importante de sus vidas y debido a ello, invierten mucho de su tiempo en él. Este uso tan frecuente que hacen del teléfono celular es uno de los aspectos que va a influir en la creación y distribución de su jerga. Es importante recordar que este es uno de los medios por excelencia del cual los y las jóvenes se valen para permanecer en contacto no solo con sus amigos más cercanos si no también con el mundo en general.
TABLA 1.

Frecuencia del uso del celular en la población participante

\begin{tabular}{llll}
\hline $\begin{array}{l}\text { Frecuencia en el uso } \\
\text { del celular }\end{array}$ & CUP & LEOM & CTPE \\
\hline Siempre & $71 \%$ & $73 \%$ & $47 \%$ \\
\hline Casi siempre & $25 \%$ & $20 \%$ & $33 \%$ \\
\hline A veces & $4 \%$ & $7 \%$ & $20 \%$ \\
\hline Casi nunca & $0 \%$ & $0 \%$ & $0 \%$ \\
\hline Nunca & $0 \%$ & $0 \%$ & $0 \%$ \\
\hline
\end{tabular}

Al consultarle a los y las estudiantes de dónde creen que se obtienen las palabras que conforman su jerga, la mayoría considera que se originan o, más bien, se obtienen del uso que se hace en las redes sociales; otra mayoría considera que son producto de la interacción social que hacen con gente de su misma edad. De hecho, en los tres colegios participantes del estudio, los porcentajes en estas categorías fueron muy altos, tal y como se muestra en la tabla 2 . En el caso de la televisión, únicamente un $13 \%$ de los participantes del CTPE considera que es fuente de jerga adolescente y un $4 \%$ de los y las participantes del CUP piensa igual.

Lo cierto del caso es que, contrario a lo que se pensaba anteriormente, la televisión dejó de ser parte de la vida de los y las jóvenes en la actualidad. Es importante recalcar que, en los conversatorios grupales, los y las participantes del CUP consideran la televisión como un medio obsoleto. Es más, al consultarle a los y las participantes acerca de la frecuencia con la que ven televisión, la mayoría confiesa que a veces lo hace, tal y como se presenta en la tabla 3. El grupo que parece hacer más uso de la televisión es el de estudiantes del CTPE, de los 
cuales un $33 \%$ dice ver televisión siempre y un $53 \%$ dice hacerlo a veces. Curiosamente, este mismo grupo de estudiantes es el que asegura que parte de su jerga la toman de la televisión. Sin embargo, lo que parece sí ser una constante aún es el hecho de que es la interacción humana la que va a producir y distribuir el léxico en un grupo social. Los mismos participantes aseguran que muchas de las palabras las escuchan de sus compañeros o de sus grupos de pares, incluso, un grupo de estudiantes del CUP asegura que en algunas ocasiones son ellos y ellas mismas las que crean o inventan los términos y comienza a darles uso. Si a esto sumamos el hecho de que los y las participantes del estudio hacen un uso muy frecuente del teléfono celular para interactuar entre sí, podemos pensar que este es un medio idóneo para la trasmisión de su jerga.

Continuando con el tema de la televisión, a pesar de que la mayoría de los y las participantes asegura no ver televisión muy a menudo, los que aseguran hacerlo confiesan que básicamente lo que ven son películas y series. Es interesante además el hecho de que los y las estudiantes del CUP aseguran ver series y películas pero lo hacen en Internet a través de sus computadoras, tablets o teléfonos inteligentes. Parte de lo que llama la atención también es el hecho de que varios de los y las estudiantes manifiestan ver noticieros, ese aspecto no es del todo relevante para este estudio pero sí es curioso el ver cómo las nuevas generaciones se preocupan por informarse acerca del acontecer nacional e internacional.

Con respecto a la radio, un $69 \%$ de la población entrevistada dice no hacer uso de este aparato y el restante $31 \%$ que confiesa escuchar radio dice hacerlo a veces. Podemos pensar entonces que estamos ante una generación totalmente diferente a la que teníamos hace $15 \mathrm{o}$ 10 años. La era digital y el internet le han ganado la batalla a la televisión y a la radio en cuanto a la preferencia del público adolescente.
TABLA 2.

Medio del cual se obtienen las palabras de la jerga adolescente

\begin{tabular}{llll}
\hline $\begin{array}{l}\text { ¿De dónde se obtiene el } \\
\text { lenguaje adolescente? }\end{array}$ & CUP & LEOM & CTPE \\
\hline Televisión & $4 \%$ & $0 \%$ & $13 \%$ \\
\hline Radio & $4 \%$ & $0 \%$ & $0 \%$ \\
\hline Redes sociales & $71 \%$ & $80 \%$ & $87 \%$ \\
\hline $\begin{array}{l}\text { Interacción con gente de } \\
\text { su misma edad }\end{array}$ & $75 \%$ & $73 \%$ & $73 \%$ \\
\hline
\end{tabular}

TABLA 3.

Frecuencia con la que los y las jóvenes ven televisión

\begin{tabular}{llll}
\hline $\begin{array}{l}\text { Frecuencia con la } \\
\text { ven televisión }\end{array}$ & CUP & LEOM & CTPE \\
\hline Siempre & $8 \%$ & $13 \%$ & $33 \%$ \\
\hline Casi siempre & $4 \%$ & $27 \%$ & $7 \%$ \\
\hline A veces & $46 \%$ & $53 \%$ & $53 \%$ \\
\hline Casi nunca & $38 \%$ & $7 \%$ & $7 \%$ \\
\hline Nunca & $4 \%$ & $0 \%$ & $0 \%$ \\
\hline
\end{tabular}

Debido al hecho de que muchos de los y las participantes consideran que el lenguaje adolescente se obtiene del uso de redes sociales, se les consultó acerca de la cantidad y el tipo de redes sociales en las cuales tienen una cuenta activa, a aprtir de ello, se obtiene que todos los grupos tienen gustos e intereses muy variados $\mathrm{y}$ esto se ve reflejado en el tipo de red social que prefieren (ver tabla 4). Por ejemplo, en el caso de los y las estudiantes del CUP la gran mayoría (83\%) dice tener una cuenta activa en la red social Snapchat y un $79 \%$ dice tener una cuenta activa en Twitter. En el caso de los y las estudiantes del LEOM, un 60\% tiene una cuenta activa en la red social Facebook. Finalmente, 
los y las participantes del CTPE poseen cuentas activas en Facebook (40\%) y en Snapchat (40\%). A pesar de ello, en relación con el uso real que hacen de las redes sociales, tal y como se aprecia en la tabla 5, la mayoría de los y las estudiantes del LEOM y el CTPE, 93\% en ambos casos, dice hacer uso más frecuente del Facebook, en tanto que el $50 \%$ de los y las estudiantes del CUP dice usar el twitter con mayor frecuencia. La red que usan con menor frecuencia es el Facebook (solo un 8\%). Este hecho es particularmente interesante debido a que el uso de las redes sociales va a determinar el tipo de léxico que usan los y las participantes del estudio. Por ejemplo, en el caso de la población de estudiantes del CUP se determina que hacen uso de acrónimos que se derivan de las etiquetas o hashtags de Twitter, entre ellos se mencionan palabras como "YALA", "YOLE", "ANLI", "ASUMLI", entre otros. Estas palabras se encuentran ausentes del léxico de los y las estudiantes del LEOM y del CTPE, pues estos hacen muy poco uso de la red social ya mencionada.

TABLA 4.

Redes sociales en las cuales los y las participantes tienen cuentas activas.

\begin{tabular}{llll}
\hline $\begin{array}{l}\text { Redes sociales en las que } \\
\text { tienen una cuenta activa }\end{array}$ & CUP & LEOM & CTPE \\
\hline Facebook & $25 \%$ & $60 \%$ & $40 \%$ \\
\hline Twitter & $79 \%$ & $27 \%$ & $20 \%$ \\
\hline Instragram & $58 \%$ & $33 \%$ & $33 \%$ \\
\hline Snapachat & $83 \%$ & $27 \%$ & $40 \%$ \\
\hline
\end{tabular}

TABLA 5.

Redes sociales usadas con más frecuencia por la población participante

\begin{tabular}{llll}
\hline $\begin{array}{l}\text { Redes social } \\
\text { de uso más } \\
\text { frecuente }\end{array}$ & CUP & LEOM & CTPE \\
\hline Facebook & $8 \%$ & $93 \%$ & $93 \%$ \\
\hline Twitter & $50 \%$ & $0 \%$ & $0 \%$ \\
\hline Instragram & $0 \%$ & $0 \%$ & $7 \%$ \\
\hline Snapachat & $42 \%$ & $7 \%$ & $7 \%$ \\
\hline
\end{tabular}

En relación con las razones por las cuales los y las participantes dicen hacer uso de la jerga adolescente, destaca el hecho de que el uso de esta les va a facilitar el proceso de identificación con otros jóvenes, un $74 \%$ de los y las participantes ve en el uso de la jerga la posibilidad de estrechar lazos con su grupo de pares, en tanto que un $17 \%$ considera que hacen uso de la jerga ya que es importante para estar a la moda, y esto les permite hacerlo. Por último, un $13 \%$ de los y las participantes del estudio dicen hacer uso de la jerga juvenil con el objetivo de que las personas adultas no entiendan las cosas que están diciendo, lo usan como una especie de código secreto que les permite ocultar información a personas que no pertenecen a su círculo de amigos. Estos datos nos dicen que, en efecto, los y las adolescentes hacen uso de su jerga para lograr esa pertenencia a los grupos de pares y con ello sentirse aceptados. Por otra parte, también se manifiesta la necesidad que tiene este tipo de población por diferenciarse del estándar $y$, particularmente, de la población adulta que en la mayoría de los casos son sus padres. 
Finalmente, se le consulta a los y las estudiantes participantes del estudio en cuál contexto consideran que ellos y ellas hacen uso de su jerga. En la mayoría de los casos, un $75 \%$ del CUP, un $100 \%$ del LEOM y un $87 \%$ en el CTPE, dicen hacer uso de su jerga adolescente en el colegio. Como segunda opción, se menciona el uso que hacen de la jerga en la calle con sus amigos. El contexto en el cual se hace menos uso de la jerga, según confiesan los y las adolescentes, es en sus casas con su familia. Algo interesante es el hecho de que el $69 \%$ de los y las participantes dicen modificar la manera en que hablan, dejan de hacer uso de su jerga dependiendo del contexto en el que se encuentren, esto con el fin de que sus interlocutores comprendan el mensaje que transmiten o simplemente con el afán de sonar cultos y educados. El $31 \%$ que dicen nunca modificar su registro considera que no tienen por qué hacerlo, ya que ellos y ellas son los mismos donde sea que estén, evidentemente nos encontramos ante un acto de rebeldía adolescente pero que, al mismo tiempo, nos deja en claro que estos jóvenes se aferran a su identidad y que no están en la disposición a cambiarla.

\subsection{Percepción de los y las adolescentes acerca del lenguaje adulto.}

Al consultarles a los y las participantes del estudio acerca de su percepción en relación con su lenguaje y el que usan personas de otras edades, se obtiene que un $78 \%$ de estos considera que su lenguaje es diferente al de los adultos y que varía particularmente en términos del vocabulario del cual hacen uso.

En cuanto a su percepción acerca del lenguaje adulto, un $50 \%$ de los y las estudiantes considera que es interesante, un 30\% lo encuentra aburrido y un $20 \%$ piensa que no hay diferencia entre el lenguaje adolescente y el lenguaje de la población adulta. Cuando se hace la discusión con los grupos de estudiantes y se les consulta al respecto, muchos consideraron que el lenguaje adulto es más culto y que generalmente no escuchan a los adultos a su alrededor hacer uso de malas palabras o de algún tipo de jerga. Sin embargo, sí confiesan que en ocasiones han escuchado a sus padres y madres hacer uso de ciertas frases o palabras cuando se encuentran en compañía de sus amigos. Consideran, además, que la clase social de las personas adultas va a generar cambios en la manera en la que hablan, al igual que la ocupación que desempeñan. Es interesante cómo los y las participantes del estudio relacionan la ausencia de jerga con una variante culta de la lengua, a pesar de ello, manifiestan encontrar el lenguaje adulto interesante $\mathrm{y}$, en muchos casos, la mayoría de ellos dice ser capaz de modificar su lenguaje para sonar culto, para ser parte del mundo adulto.

\subsection{Percepción de los y las adolescentes acerca de las diferencias de género en el lenguaje}

Al consultarle a la población participante si el lenguaje de los hombres es similar o diferente al de las mujeres, se logra tener una gran diversidad de criterios al respecto. En el caso de los y las estudiantes del CTPE, un $87 \%$ lo considera diferente, de la misma forma, un $93 \%$ de los y las estudiantes del LEOM ve diferencias de género en el lenguaje. Sin embargo, los y las estudiantes del CUP difieren completamente, ya que un $79 \%$ de esa población considera que no existen diferencias entre una población y otra. Las personas que consideran que existen diferencias de género en el lenguaje juvenil dicen que los hombres tienden a ser más vulgares y hacer uso de un vocabulario más soez en tanto que las mujeres son más educadas y casi no hacen uso de malas palabras. Por su parte, el grupo de estudiantes del CUP, en el cual se produce una marcada diferencia de criterio en relación con los otros grupos, considera que el lenguaje masculino y femenino es igual, sin embargo, cuando se hace el conversatorio con el grupo, ellos y ellas aceptan que existen palabras que consideran más masculinas o más femeninas que otras, por ejemplo "cosi", "holis" y "chais" las perciben como palabras muy femeninas en tanto que las palabras groseras o insultos se perciben como más masculinas, a pesar de esto, las mujeres confiesan que ellas no tienen 
problemas en usarlas ya que no hay diferencias entre hombres y mujeres en términos de lenguaje y también advierten que sus compañeros hacen uso de palabras femeninas de manera sarcástica para burlarse de ellas. Los varones de este grupo consideran que si bien es cierto ellos son "mal hablados" las mujeres también lo son pero lo disimulan más. Parece entonces que a pesar de que los y las adolescentes del CUP manifiestan en su mayoría que no existen diferencias entre hombres y mujeres en términos de lenguaje, sí las hay y son ellos y ellas mismas quienes las subrayan al aceptar la existencia de palabras masculinas y femeninas. En el caso de los estudiantes de los otros dos colegios, ellos expresan que existen diferencias, las cuales coinciden con lo que diferentes autores apuntan, por ejemplo, el hecho de considerar al lenguaje de los hombres más soez y vulgar.

\subsection{Percepción de los y las adolescentes con respecto a las diferencias lingüísticas en relación con lugar en el que viven las personas}

Cuando se le consulta a la población participante si consideran que el lenguaje que se usa en las zonas urbanas es similar o no al que se usa en las zonas rurales, la percepción de los diferentes grupos es bastante homogénea. Un $96 \%$ de los y las participantes del CUP cree que el lenguaje de las zonas rurales es diferente al de las zonas urbanas, un $100 \%$ de los y las estudiantes del LEOM piensa de la misma forma $\mathrm{y}$, de igual manera, lo hace el $93 \%$ de los y las estudiantes del CTPE. En esta misma línea, al consultarle al estudiantado si consideran que el lugar en donde vive una persona tiene influencia en su forma de hablar, un $80 \%$ de los y las estudiantes del CTPE considera que sí, un $87 \%$ de los y las estudiantes del LEOM piensa de la misma forma, al igual que el $96 \%$ de los y las participantes del CUP.

En este punto es donde el prestigio lingüístico toma relevancia. Al hablar con los y las participantes del CUP y el LEOM, ellos confiesan que el lenguaje que se usa en las zonas rurales es menos educado, es de cierta forma más "acampesiado" o "maicero". Los y las estudiantes del CTPE, por su parte, consideran que las personas de Puntarenas (zona urbana) hablan mal, ya que son más "pintas", en tanto que las personas de las zonas rurales son más humildes y más educados. Podemos ver entonces cómo cada grupo defiende su variante léxica $y$, de cierta forma, desprestigia las otras. En lo que sí están de acuerdo todos los grupos es en decir que las personas de clase más alta tienen un habla más educada y culta que las personas de clase más baja; se ve entonces cómo se le da un prestigio a la clase más alta aunque no sea necesariamente la más educada. Por ejemplo, un estudiante se refirió al hecho de que las personas de "El progreso", que es un barrio de clase baja y con mucha problemática de tipo delictiva hablan peor que sus compañeras (que no viven en esta zona).

\section{Conclusiones}

El lenguaje adolescente es una constante en donde sea que existan grupos de personas en esa etapa de la vida, este les da identidad, les facilita la posibilidad de estrechar su relación con sus pares y con ello construir su personalidad.

La jerga define a un grupo, independientemente de su edad, género, estrato social o localización geográfica. En el caso de los y las adolescentes, les permite diferenciarse de las normas y los estándares sociales, particularmente, es una forma de alejarse, hasta cierto punto, del control que pueden ejercer sus padres sobre ellos. El solo hecho de ser capaces de usar palabras o frases que sus progenitores no puedan comprender les da poder sobre estos y ello es un aspecto que los y las adolescentes en medio de su rebeldía valoran mucho.

Los y las jóvenes, al igual que cualquier otro ser humano, poseen la capacidad de modificar su registro en el momento y lugar en que lo estimen conveniente y estos cambios obedecen a muchas razones: en primera instancia, la jerga adolescente no cuenta con un prestigio lingüístico alto para la comunidad en general a pesar de que para los jóvenes posea un prestigio encubierto. Se entiende el lenguaje 
estándar como culto y educado, en tanto que el lenguaje juvenil no se valora de esta forma, sin embargo, eso es algo que tiene sin cuidado a los y las jóvenes que lo usan, probablemente esa es la razón por la cual el contexto en el cual esta variante lingüística se usa más sea en el colegio, puesto que es un lugar seguro para ellos y ellas, es un sitio en el cual sus similares pueden entender lo que dicen y, además, , son personas que pasan por las mismas situaciones que ellos y ellas. Es importante entender que los y las jóvenes pasan más de la mitad de su día en el colegio y gran parte del día se mantienen en constante interacción con su grupo de pares a través de los medios tecnológicos. La interacción con personas de su misma edad, según los y las participantes de este estudio, es una clave para la creación y reproducción de la jerga adolescente. Cuando los y las adolescentes están juntos abren de manera más libre sus pensamientos y es ahí en donde se acuñan muchos términos que en algunos casos forman parte del banco de palabras de esta población por algún tiempo y, en otros casos menos comunes, dichas palabras salen de los grupos de jóvenes y se integran al vocabulario de otras personas.

La televisión y la radio ya no son entes generadores del léxico adolescente como lo eran hace varios años; los y las jóvenes ya no consumen televisión de la forma en que lo hacían las generaciones pasadas. En la actualidad lo que realmente mueve a la población juvenil, al menos a la que participa en este estudio, son los medios digitales, particularmente el teléfono celular. Parece ser que los y las jóvenes de zonas rurales y de estratos sociales más bajos mantienen hasta cierto punto la costumbre de ver televisión, esto probablemente debido a que no cuentan con tantos recursos como para sustituir este aparato por otros como una computadora con acceso a Internet.

Las películas y series son los programas favoritos de los y las adolescentes de este estudio, probablemente esto explica el uso de anglicismos dentro de su jerga. En relación con los anglicismos, es posible que se estén obteniendo del uso que los y las jóvenes hacen de las redes sociales.
Las redes sociales son populares entre los y las jóvenes y las encuentran muy eficaces como medio de comunicación. De hecho, algo que llama la atención, al menos en los y las estudiantes del CUP, es que la mayoría de esta población hace uso de la red social Twitter, aunque no goza de mucha popularidad entre la población adulta de Costa Rica, por ende, ellos y ellas saben que sus padres difícilmente los van a encontrar ahí y aunque lo hagan, ellos hacen uso de ciertos códigos que va a dificultar el nivel de comprensión de sus mensajes por parte de la población adulta.

Con respecto a las variaciones léxicas entre personas de distintos grupos etarios, los y las participantes ven claras diferencias entre el lenguaje que usan ellos y ellas y el de las personas adultas, particularmente en términos de léxico; nuevamente, se aprecia el lenguaje adulto como culto, hecho que le da prestigio, sin embargo, es claro que a los y las adolescentes no les interesa el prestigio lingüístico, pues a pesar de considerar ciertas variaciones más prestigiosas que las que ellos y ellas usan, no se interesan por hacer uso de estas salvo en ocasiones específicas.

Existe además una marcada diferencia, al menos en términos de percepción, entre el habla de las zonas rurales y las zonas urbanas. Las personas de zonas urbanas atribuyen las diferencias a la falta de oportunidades y limitaciones tecnológicas que poseen las personas de las zonas rurales, en tanto que los y las jóvenes que habitan en zonas rurales consideran el lenguaje de zonas urbanas más fuerte y vulgar, eso sin dejar de aceptar que el lenguaje de las zonas rurales no es del todo culto.

El lenguaje masculino y femenino se percibe como diferente, esto nos muestra, tristemente, que los estereotipos sociales continúan. Por ejemplo, los y las jóvenes siguen pensando que los hombres tienen un vocabulario más vulgar que las mujeres y en realidad eso no es del todo cierto, de la misma forma, se les atribuye a las mujeres el uso de un lenguaje que se visualiza como soso y hasta tonto. Hemos mantenido ese tipo de criterios por años y lo hemos heredado a las nuevas 
generaciones, prueba de ello es lo que este grupo de participantes manifiesta.

Finalmente, tenemos una variación léxica que existe en la población adolescente, la cual se ve potenciada por los medios tecnológicos y la interacción de los grupos de pares. Existe una percepción clara en relación con las diferencias de género, edad y el lugar del cual las personas provienen o el lugar en el que viven. La jerga adolescente cambiará mucho con el paso del tiempo, sin embargo, es importante entender los procesos que lo mueven y la percepción de la población que hace uso de este.

\section{Referencias}

Aguilar, W, Pérez, R y Víquez, D. 2007. El periodo juvenil visto desde la perspectiva adulta. Revista Ciencias Sociales, 116: 15-33. Recuperado de http://revistas. ucr.ac.cr/index.php/sociales/article/ viewFile/11033/10406

Arroyo, L, Huertas, P, Peirano, Cy Pérez, M. 2014. La identidad del adolescente y su relación con el imaginario nacional costarricense. Revista Actualidades Investigativas en Educación, 14 (2): 1-32. doi: http://dx.doi. org/10.15517/aie.v14i2.14798_

Boyer, J. 2013. Forma y función de los juegos fónicos en el habla juvenil de Puebla (México) (Tesis de maestría, Université de Montréal, Montreal, Canadá). Recuperado de https://papyrus.bib.umontreal.ca/ xmlui/bitstream/handle/1866/11001/ Boyer_Julie_2013_memoire.pdf

Chang, M y Guadalupe, J. 2013. El lenguaje cotidiano de los alumnos universitarios. Recuperado de http://cenedic.ucol.mx/ fieel/2013/ponencias_pdf/26.pdf

García, R. 2004. Juventud y medios de comunicación. La televisión y los jóvenes: aproximación estructural a la programación y los mensajes. Inámbitos, 11 (12): 115-129. Recuperado de http:// www.ull.es/publicaciones/latina/ ambitos/11-12/archivos11_12/reina.pdf

Grinder, R. 1976. Adolescencia. Editorial Limusa, México.

Krauskopf, D. 2006. Adolescencia y educación. $2^{\mathrm{a}}$ edición, Editorial UNED: San José.

Mitkova, A. 2009. El estilo comunicativo de los jóvenes. CÍRCULO de Lingüística Aplicada a la Comunicación (clac), 37: 21-44. Recuperado de http:// pendientedemigracion.ucm.es/info/ circulo/no37/mitkova.pdf

Molina, G y Toledo, K. 2014. Las redes sociales y su influencia en el comportamiento de los adolescentes, estudio a realizarse en cuatro colegios de la ciudad de Cuenca con los alumnos de primero año de bachillerato (Tesis de licenciatura). Recuperado de http://dspace.uazuay.edu. ec/bitstream/datos/3659/1/10335.PDF

Pérez, R. 2002. Televisión, juventud y futuro: estudio intercultural con jóvenes costarricenses y alemanes. Revista Actualidades en Psicología, 18 (105): 7-24. Recuperado de http://revistas.ucr.ac.cr/ index.php/actualidades/article/view/44/47

Ríos, P. 2010. Comunicación móvil: el uso del celular en la relación entre madres $e$ hijos adolescentes (Tesis de maestría). Recuperado de http://www.flacso.edu. $\underline{\mathrm{mx} / \text { biblioiberoamericana/TEXT/MCS }}$ XVII_promocion_2008-2010/Rios_P.pdf

Rodríguez, F. 2006. Medios de comunicación y contracultura juvenil. CÍRCULO de Lingüística Aplicada a la Comunicación (clac), 25: 5-30. Recuperado de http:// pendientedemigracion.ucm.es/info/ circulo/no25/rodriguez.pdf 
Rundblom, M. 2013. Un estudio del lenguaje soez entre jóvenes en Madrid. ¿Hay diferencias entre géneros? Recuperado de http://www.divaportal.se/smash/get/ diva2:645651/FULLTEXT01.pdf

Sádaba, C. 2005. Instrucción: juventud y medios de comunicación frente a frente. Revista Juventud. 68: 9-18. Recuperado de http://www.injuve.es/sites/default/files/ revista68_1.pdf

Salazar, A. 2015. El prestigio frente a la identidad: las actitudes lingüísticas de los monterianos hacia el español hablado en Montería. Cuadernos de Lingüisticas Hispánica, 25: 39-55. Recuperado de http://www.scielo.org.co/pdf/clin/n25/ n25a03.pdf

Sánchez, V y Ramírez, N. 2008. Aspectos morfológicos y cambios de forma en la creación léxica de la jerga de la Sede Guanacaste, Universidad de Costa Rica. Revista Káñina, 32 (2): 93-100. Recuperado de http://revistas.ucr.ac.cr/ index.php/kanina/article/view/4113/3939
Valeš, M. 2009. Patrones de prestigio y cambio lingüístico en Granada. Études romanes de Brno, 30 (2): 231-239. Recuperado de https://digilib.phil.muni.cz/bitstream/ handle/11222.digilib/114809/1 EtudesRomanesDeBrno_39-2009-2_27. pdf

Velázquez, A. 2007. Lenguaje e identidad en los adolescentes de hoy. Revista El Ágora USB, 7 (1): 85-107. Recuperado de http://web.usbmed.edu.co/usbmed/ elagora/docs/vol7nro1/Catedra\%20 Abierta\%20\%28LENGUAJE\%20E\%20 IDENTIDAD $\% 20$ EN $\% 20 \mathrm{LOS} \% 20$ A D O L E S C E N T E S \% 20 DE \% 20 HOY\%29\%207.pdf

Yule, George. 2010. The study of language. 4th edition. New York. Cambridge University press.

Zimmer, T. 2004. El lenguaje estudiantil de Costa Rica: el disfemismo como medio de identificación. Revista Káñina, 28 (2): 161-178.Recuperado de http://revistas. ucr.ac.cr/index.php/kanina/article/ view/4730/4544

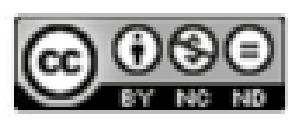

Este obra está bajo una licencia de Creative Commons Reconocimiento-NoComercial-SinObraDerivada 4.0 Internacional. 